Revue d'histoire de l'Amérique française

ZWB REVUE D.HISTOIRE DE L'AMÉRIQUE FRANÇAISE

\title{
Un cadet de Gascogne : Philippe de Rigaud de Vaudreuil
}

\section{Guy Frégault}

Volume 5, numéro 1, juin 1951

URI : https://id.erudit.org/iderudit/801683ar

DOI : https://doi.org/10.7202/801683ar

Aller au sommaire du numéro

Éditeur(s)

Institut d'histoire de l'Amérique française

ISSN

0035-2357 (imprimé)

1492-1383 (numérique)

Découvrir la revue

Citer cet article

Frégault, G. (1951). Un cadet de Gascogne : Philippe de Rigaud de Vaudreuil. Revue d'histoire de l'Amérique française, 5(1), 15-44.

https://doi.org/10.7202/801683ar d'utilisation que vous pouvez consulter en ligne.

https://apropos.erudit.org/fr/usagers/politique-dutilisation/ 


\section{UN CADET DE GASCOGNE: PHILLIPE DE RIGAUD DE VAUDREUIL}

En 1750, madame Bégon écrit à son gendre, le commissaire-ordonnateur Michel: "Tu as bien raison de dire qu'il n'y a rien d'aussi heureux que la famille Vaudreuil. Je vois de tout côté qu'ils réussissent à tout ce qu'ils entreprennent." 'La sympathique épistolière n'exagère pas. La f: mille de Vaudreuil connaît de beaux jours. L'aîné, Louis-Philippe, naguère comte et maintenant marquis, s'est couvert de gloire au cours de la dernière guerre; il est maintenant chef d'escadre; d'autres honneurs l'attendent, et, dans trois ans, il sera promuflieutenant général des armées navales. Jean, le vicomte, a pris part à maintes campagnes; au siège de Prague, il a perdu son frère, le baron Philippe-Antoine, qui, après avoir pensé entrer dans les ordres, s'était engagé brillamment dans la vie des camps; le vicomte a été major général de l'armée de Bohème, puis major général de l'armée des Flandres; il a vu la journée de Fontenoy; le voici lieutenant général des armées du roi depuis 1748 et major aux Gardes Françaises; Louis XV l'a décoré du cordon rouge en attendant de le recevoir sous peu grand-croix de l'ordre de Saint-Louis. Pierre occupe avec éclat le poste de gouverneur de la Louisiane. François-Pierre, que les Canadiens appellent monsieur de Rigaud, s'est révélé officier d'un grand mérite, il possède toutes les qualités d'un chef $^{2}$; aussi, en récompense de ses services, le roi l'a-t-il nommé gouverneur des Trois-Rivières. Le cadet, Joseph-Hyacinthe, poursuit aux Antilles une carrière étonnante; il se querelle, intrépide, avec les plus hauts personnages ${ }^{3}$; protégé du maréchal de Noailles et du duc d'Ayen,

1. Lettre du 15 septembre 1750, Rapport de l'Archiviste de la province de Québec [ RAPQ] pour 1984-1935 (Québec, 1935), 114.

2. Hocquart a Maurepas, 6 octobre 1746, Archives des Colonies, Correspondance générale, Canada [ AC, C 11A ], 85: 375.

3. Rouillé au maréchal de Noailles, 17 novembre 1752, AC, B 96: 175 bis; id. au duc d'Ayen, 17 novembre 1752, ibid., 175 bisv; Mme Bégon à Michel, 2 janvier 1752, RAPQ (1934-1935), 163. 
il s'est vu confier le gouvernement de l'ouest et du sud de SaintDomingue; Sa Majesté l'élèvera bientôt au rang de commandant général des Iles sous les Vents. La famille est chargée de titres, comblée d'honneurs, constellée de décorations."

L'auteur de cette extraordinaire fortune a consacré toute sa longue vie à la préparer et il a eu le bonheur d'en jouir tout le premier. C'est en Amérique, où trois de ses fils détiennent maintenant trois gouvernements, que, sans hâte comme sans relâche, il a pourvu à sa propre ascension et à celle des siens. Lorsque Philippe de Rigaud de Vaudreuil débarque au Canada, en 1687, il a déjà quarante-trois ou quarante-quatre ans ${ }^{5}$, l'âge qu'aura son quatrième fils quand celui-ci sera désigné gouverneur de la Louisiane. Il débute modestement, en qualité de commandant des troupes du détachement de la marine, titre que la Cour a créé pour lui le 17 mars précédent ${ }^{6}$. Ses fonctions consistent à établir une liaison plus étroite entre les diverses compagnies qui constituent la garnison de la colonie; en pratique, il semble bien que son rôle se borne à "contenir les troupes dans une plus grande subordination par de fréquentes revues"". Le ministre supprimera cette charge en 1714. Etait-elle bien nécessaire? Il est permis d'en douter: malgré les efforts répétés de Vaudreuil lui-même pour la faire rétablir en faveur de ses fils, il n'y parviendra jamais, ${ }^{8}$

4. Sur la carrière des Vaudreuil, voir l'ouvrage un peu rapide mais généralement bien informé de Pierre-Georges Roy, La Famille de Rigaud de Vaudreuil (Lévis, 1938), passim. [ A l'avenir: Roy ].

5. Cyprien Tangưay, Dictionnaire genéalogique des familles canadiennes (7 vol., Montréal 1871-1890), 1: 184; Roy, 12. Remarquons cependant que les registres paroissiaux de la présente commune de Vaudreuille ne remontent pas plus haut que 1650 et que, par conséquent, personne n'a encore pu trouver quand exactement Philippe de Rigaud de Vaudreuil est né, Francis H. Hammang, The Marquis de Vaudreuil: New France at the Beginning of the Eighteenth Century (Bruges et Louvain, 1938), 41, note 3. [ A l'avenir: Hammang ].

6. Pierre-Georges Roy, Les Petites Choses de notre histoire (6 vol., Lévis, 19191931), 6: 91.

7. "Demandes d'emplois militaires au Canada," [ 1717 ], AC, C 11A, 37: 467. Voir Champigny à Seignelay, 16 juillet 1696, dans E.B. O'Callaghan, éd., Documents Relating to the Colonial History of the State of New York (11 vol., Albany, 1853-1887), 9: 331. [ A l'avenir, nous citerons cette compilation sous le sigle NYCD. ] Denonville à Seignelay, 25 aout 1687, ibid., 340.

8. Vaudreuil à Maurepas, 25 octobre 1724, AC, C 11A, 46: 83; Maurepas à Sabrevois, 6 juin 1724, AC, B 47: 1181; $i d$. à Vaudreuil, 5 juin 1725, AC, B 48: 873; $i d$. à Vaudreuil de Cavagnial, 5 juin 1725, ibid., 890; id. à id., 1er mai 1731, AC, B 55: 511-511v. 
et la Nouvelle-France ne s'en portera pas plus mal. En 1687, on tente donc l'expérience de nommer au Canada un officier d'état-major à l'occasion des renforts dépêchés contre les Iroquois. Le poste convient à un soldat de carrière. Le chevalier de Vaudreuil en est un. Vers 1670, il est entré dans les mousquetaires du roi, puis il a servi comme aide-de-camp dans les armées françaises en Allemagne ${ }^{9}$. Il ne paraît pas s'y ềtre signalé, mais il y a acquis de l'expérience, le respect absolu de la discipline, l'habitude de l'obéissance et l'art du commandement. Vient-il en Nouvelle-France pour avancer plus vite qu'en Europe? Son empressement à se faire valoir en donne l'impression. Si tel est son calcul, il ne se trompe point: dans la colonie, un homme de talent peut parvenir à presque tout, dès qu'il a de la naissance et de la protection. Le chevalier ne manque ni de l'une ni de l'autre.

En arrivant au Canada, il inspire pourtant quelque compassion au gouverneur général. Denonville confie au secrétaire d'Etat à la Marine, de qui relève l'administration des colonies: "C'est un cadet de calité de Gascogne qui ne fera pas venir souvent de lettres de change si vous n'avés la bonté de luy en donner les moyens... J'espère que vous aurés la bonté de le secourir."'10 Le gouverneur a raison. Vaudreuil est pauvre, sa famille éprouve de graves embarras d'argent ${ }^{11}$. Mais la plaie ne se révèle pas mortelle; avec le temps, les grâces de Sa Majesté la guériront. En vrai cadet de Gascogne, le chevalier porte un blason qui, pour n'être pas doré, n'en reste pas moins précieux. Issu d'une des plus anciennes familles du Languedoc, il peut faire remonter sa généalogie jusqu'au treizième siècle, à moins que ce ne soit jusqu'au douzième ou peut-être encore jusqu'au neuvième ${ }^{12}$. Sans doute le prestige de sa maison ne dépasse-til pas un coin de province. Qu'importe? c'est quand même le prestige

9. "J'ay entré dans les mousquetaires aussy jeune que mon ayné," Vaudreuil a Maurepas, 16 novembre 1704, RAPQ (1938-1939), 49. En 1704, Louis-Philippe, le fils aîné de Vaudreuil a treize ans. Il ne serait pas incroyable que le gouverneur eât commencé sa carrière aussi jeưne. Mais d'autres témoignages contredisent le sien, qui est d'ailleurs intéressé. Sur cette question, voir les textes produits par Hammang, 43 .

10 Denonvile à Seignelay, 28 octobre 1687, cité par Hammang, 44, note 12.

11. Hammang, 42.

12. On retrace habituellement la généalogie de Vaudreuil d'après d'Hozier, Armorial général de la France (8 vol., Paris, 1736-1768), 6: 323. 
de la vieille noblesse. Le commandant des troupes en conçoit une immense fierté et il accepte en galant homme les obligations qui en découlent. Ainsi, parce qu'elle aura été "depuis tres longtemps dans la maison de Rigaud", il n'épargnera rien pour recouvrer la terre de Vaudreuil, tombée par aventure entre des mains étrangères, et, dans son testament, il prendra les dispositions les plus minutieuses pour que, d'une génération à l'autre, cette terre passe "d'aisné masle en aisné masle... de la famille de Rigaud,... l'ordre de primogéniture garde",13. Il a comme le sentiment de tenir à une dynastie.

Il faut être homme de qualité pour parvenir régulièrement aux grands emplois militaires. Il est bon de s'assurer aussi l'appui de quelque grand. Qui protégera Vaudreuil ? Un ministre, - le ministre, disent les officiers attachés au service des colonies, - Jérôme de Pontchartrain. En 1699, le comte de Pontchartrain succède à son père, le Chancelier, dans la charge de secrétaire d'Etat. Il conservera son poste jusqu'en 1715. Entre lui et Vaudreuil, bien entendu, aucune familiarité; d'une part, une bienveillante condescendance, de l'autre, une soumission et un respect que renforcent les liens de la reconnaissance. Pontchartrain parle en maître. A l'occasion, il gourmande son protégé; celui-ci commet-il une bévue, le secrétaire d'Etat lui donne sur les doigts, lui faisant entendre de prendre garde "de tomber à l'advenir dans de pareils inconvenients", parce qu'alors il serait impossible d'en celer la connaissance au roi ${ }^{14}$. Quand Vaudreuil atteindra sa suprême ambition, il déclarera au ministre: "Je vous ay l'entiere obligation de l'honneur que Sa Majesté m'a fait de m'accorder le gouvernement general de ce pays"'15. Et rien ne sera plus vrai. Pontchartrain ne laisse pas passer l'occasion de souligner qu'il s'intéresse au gentilhomme du Languedoc ${ }^{16}$. A mesure que les années s'écoulent, son intérêt s'accroît. "Vous connoissez les sentimens que j'ay pour vous", affirme-t-il à Vaudreuil, en 1712, "et combien

13. Voir une reproduction photographique du testament de Vaudreuil, 10 novembre 1718, dans Pierre-Georges Roy, $L a$ Ville de Québec sous le régime français (2 vol., Québec, 1930), 2: entre 101-102.

14. Pontchartrain à Vaudreuil, 9 juin 1706, AC, B 27: 225v.

15. Vaudreuil à Pontchartrain, 16 novembre 1701, AC, C 11A, 22: 49; ibid., 65. Voir Pontchartrain a Vaudreuil, 14 juin 1704, AC, B 25: $87 \mathrm{v}$.

16. Pontchartrain a Basville, 5 septembre 1709, Archives de la Marine [ AM ], B 2, 208: 1124-1125; id d̀ id., 10 mars 1715, AC, B 37: 45v. 
je suis porté a vous rendre service, vous pouvez compter aussy que le Roy aura soin de vôtre famille et je vous exhorte d'estre tranquille sur cela et d'estre persuadé que j'auray toujours toute l'attention que vous pouvez desirer pour ce qui vous appartient"17. L'année suivante, il lui répète: "Vous ne devez pas douter de l'envie que j'ay de vous faire plaisir... ${ }^{18 "}$ En 1714, les mêmes mots reviennent sous sa plume: "Je vous feray toujours et à vôtre famille tous les plaisirs qui pourront dependre de moy"19". Vaudreuil ne peut rêver d'un plus puissant soutien. Viennent les années de la Régence, Pontchartrain tombera, mais, en 1723, c'est son fils, Maurepas, qui prend le portefeuille de la Marine. Le père a fait la carrière de Vaudreuil, le fils fera celles des fils de Vaudreuil. Le cadet de Gascogne aura eu de la chance.

Il n'aura pas, toutefois, attendu la fortune sur le pas de sa porte. Sitôt qu'il arrive à Québec, il sollicite l'honneur d'aller se battre aux côtés de Denonville contre les Tsonnontouans ${ }^{20}$. Au retour de l'expédition, le gouverneur le met à la tête d'un détachement d'une centaine de coureurs de bois, - pardon, de "mousquetaires", corps mobile, destiné à couvrir Montréal durant l'hiver'. En 1688, Callière va exposer à la Cour les plans qu'il a élaborés en vue de l'invasion de la Nouvelle-York ${ }^{22}$; en son absence, Vaudreuil, sur l'ordre du gouverneur général, assure l'intérim au gouvernement de Montréal ${ }^{23}$. L'année suivante, le roi se déclare satisfait des services du chevalier et, sans lui enlever son titre de commandant des troupes,

17. Pontchartrain à Vaudreuil, 18 juin 1712, AC, B 34: 28.

18. Id à $i d$., 4 juillet 1713, ibid., 35: 109-109v.

19. Id. à id., 24 mai 1714, ibid., 36: 411.

20. Denonville a d'abord songé à laisser Vaudreuil a l'intérieur pour assurer la sécurité des habitants durant la campagne. Denonville à Seignelay, 8 juin 1687, NYCD, 9: 328.

21. Collection de manuscrits contenant lettres, mémoires et autres documents historiques relatifs à la Nouvelle-France (4 vol., Québec, 1883-1885), 1:571s. [ A l'avenir: Coll. de Mss. ] Sur cette expédition, voir le mémoire de Denonville, octobre 1687, NYCD, 9: 358-369.

22. Les divers mémoires de Callière sur la prise de New-York sont dans $\mathbf{A C}$, C 11A, 10: 260-278v; ils ont été imprimés dans le Bulletin des recherches historiques [ BRH], 24 (1918): 289-302, 321-329, 353-367.

23. Coll. de Mss., 1: 567. 
il le fait capitaine d'une compagnie régulière ${ }^{24}$. Il y a plus. En France, aidé par la tournure que prennent les événements d'Angleterre ${ }^{25}$ le gouverneur de Montréal a réussi à convaincre le ministre qu'il faut envahir New-York. Frontenac revient en Amérique avec l'ordre de diriger l'opération, assisté de Callière, auteur des plans de campagne $^{26}$. Les chefs partis, à qui reviendra le commandement de la colonie? La Cour veut que ce soit à Vaudreuil ${ }^{27}$. Voilà donc celui-ci élevé au troisième rang parmi les officiers supérieurs de la NouvelleFrance.

En 1689, cependant, loin de pouvoir prendre l'offensive, le Canada se voit terrassé par le massacre de Lachine, paralysé par la terreur. Comme d'autres, plus que d'autres, Vaudreuil se sent impuissant devant tant d'horreur. Chargé par Denonville de lancer une contreattaque sur l'ennemi retranché en haut de l'île de Montréal, il retient ses troupes au moment où elles vont traverser un bois pour déboucher sur le camp des Iroquois; les trois quarts de ceux-ci dorment pourtant, "morts yvres des eaux de vie qu'ils avoient pris chez les habitans", l'occasion s'offre d'en "tuer cinq ou six cents"28. Vaudreuil recule, pendant que Subercase, méprisant, en vient avec lui aux gros mots. Il se reprend quand Sir William Phips vient mettre le siège devant Québec en octobre $1690^{29}$. Plus de vingt ans après, Ruette d'Auteuil accusera le chevalier de s'être conduit lâchement en pré-

24. "Instruction pour le sieur comte de Frontenac," 16 juin 1689, RAPQ (1927-1928), 7.

25. Louis XIV déclare la guerre "à l'usurpateur d'Angleterre" le 25 jưin 1689, "Ordonnance du Roy portant declaration de guerre," Coll. de Mss., 1: 463.

26. "Mémoire pour servir d'instruction à monsieur le comte de Frontenac sur l'entreprise de la Noưvelle-York," 7 juin 1689, RAPQ (1927-1928), 12-16. Sur cet épisode, voir la brillante étưde de Marcel Trudel, "Lou'is XIV et son projet de déportation, 1689", Revue d'histoire de l'Amérique française, 4 (1950): 157-171.

27. RAPQ (1927-1928), 13; Seignelay à Frontenac, 7 juin 1689, ibid., 16; Seignelay à Denonville, 7 juin 1689 , Coll. de Mss., 1: 462; "Ordre du roi au sieưr chevalier de Vaudreuil pour commander au pays de Canada en l'absence des Srs comte de Frontenac et chevalier de Callières," 7 ju in 1689, BRH, 26 (1919): 14.

28. Coll. de Mss., 1: 569; Vachon de Belmont, Histoire du Caniada, 30, dans Société littéraire et historiquie de Québec, Collection de mémoires et de relationis sur l'histoire ancienne du Canada (Québec, 1840).

29. "Relation de Monseignat," dans Ernest Myrand, éd., Sir William Phips devant Québec (Québec, 1893), 22, 24. Le secrétaire de Frontenac donne ici à Vaudreuil le grade de "colonel des troupes". 
sence des Bostonnais. Mais l'ancien procureur général est un dénigreur $^{30}$.

L'invasion repoussée, le pays est en liesse ${ }^{31}$. Le commandant des troupes choisit ce moment pour se marier. Le 21 novembre 1690, l'évêque célèbre lui-même la cérémonie en présence du gouverneur général ${ }^{32}$. Louise-Elisabeth de Joybert a dix-sept ans, trente de moins que Vaudreuil. Fille de Pierre de Joybert, sieur de Marson, commandant à Gemseck sur la rivière Saint-Jean, elle est née en Acadie le 18 août 1673 ; on l'a tout de suite ondoyée, quitte à la faire baptiser solennellement à Québec deux ans plus tard: si le comte de Frontenac a bonne mémoire, il se rappelle l'avoir tenue sur les fonts baptismaux ${ }^{33}$. L'enfant a connu une existence agitée. Elle n'avait pas encore cinq ans lorsque son père, qui s'était copieusement querellé avec son chef, est mort après s'être fait enlever et rançonner par les Anglais ${ }^{34}$. Marson n'a rien laissé qu'un fief perdu à l'autre bout de l'Amérique française. Sa veuve s'est bien fait octroyer une gratification annuelle de 600 livres, mais le ministre a d'abord négligé de la lui verser ${ }^{35}$, puis il l'a réduite de moitié. En 1685, elle fait pitié: "Il y a encore icy, écrit Denonville, une pauvre Demoiselle, veuve du Sieur Marson qui a esté major dans l'Acadie qui fut pillée par les Anglois de Baston. Elle est réduitte, Monseigneur, avec ses enfants, à n'avoir pas de pain ${ }^{36}$ ". La filleule de Frontenac n'en a pas

30. Il raconte que Vau'dreuil abandonna ses hommes sous le feu de l'ennemi "et se retira seul à Québec chercher les reproches de Mr de Frontenac", "Mémoire sur l'état présent du Canada," 1712, RAPQ (1922-1923), 45. Frontenac, croyons-nous, n'eât pas manqué de flétrir une pareille conduite, et si Auteuil disait vrai, Monseignat eat parlé autrement qu'il n'a fait de Vaudreuil; le gơuverneur a, du reste, endossé la "relatión" de son secrétaire, Frontenac à Seignelay, 12 novembre 1690, RAPQ (1927-1928). 40. Vaudreuil range Ruette d'Auteuil au nombre de ses "plus eruels ennemis", Hammang, 71, note 115.

31. Coll. de Mss., 1: 578.

32. On trouvera une reproduction photographique de l'acte de mariage de Vaudreuil dans P.-G. Roy, La Ville de Québec sous le régime français, 2: entre 24 et 25.

33. Ibid., 1: 541. Voir Ernest Gagnon, Le Fort et le Chateau Saint-Louis (Montréal, 1925), 70 .

34. Fróntenac à Colbert, 2 novembre 1672, RAPQ (1926-1927), 17; "Mémoire sur le Canada," Coll. de Mss., 1: 243; Frontenac à Colbert, 14 novembre 1674, RAPQ (1926-1927), 73s; id à id., 6 novembre 1679, ibid., 111.

35. Résumé des lettres de de Meulles, 1682, Coll. de Mss., 1: 290.

36. Denonville à Seignelay, 13 novembre 1685, ibid., 346. 
moins reçu son instruction chez les Ursulines, qui lui ont montré à tirer des révérences, mais point du tout à mettre l'orthographe ${ }^{37}$. C'est une jeune personne de qualité, "douée de toutes les grâces qui font le charme d'un cercle d'elite"; on la peint sage et modeste, et sa figure a des "attraits" 38 . Un lien très fort se noue entre Vaudreuil et le Canada.

Depuis le siège de Québec jusqu'à la fin de la guerre de la Ligue d'Augsbourg, - en Amérique, on dit la guerre du roi Guillaume, les années se succèdent glorieuses pour la Nouvelle-France. C'est l'époque des campagnes d'Iberville. Pendant que le Cid canadien poursuit les Anglais sur terre et sur mer, a la baie d'Hudson, en Acadie et à Terre-Neuve, Vaudreuil rencontre à plusieurs reprises les Iroquois. En 1691, à Repentigny, il remporte sur les Goyogoins une importante victoire qui permet aux habitants du gouvernement de Montréal de faire leurs semences ${ }^{39}$. L'année suivante, il écrase au Long-Sault une bande de 200 Iroquois qui avaient eu l'audace de se répandre jusqu'au-dessous de l'île de Montréal; en racontant cette brillante affaire, le gouverneur ne peut taire son admiration: "C'est, écrit-il du chef de l'expédition, un gentilhomme de beaucoup de valeur et de très bonne volonté" ${ }^{40}$. En 1696, le chevalier accompagne Frontenac au cœur du territoire ennemi et se signale par la "diligence incroyable" avec laquelle il saccage le village des Onneyouts et détruit leurs moissons ${ }^{41}$.

Entre deux campagnes, des affaires de famille le rappellent parfois en France, où jamais ses séjours ne sont inutiles à son avancement. Il y passe une première fois en 1692 et rentre dans la colonie

37. Voir Mme de Vaudreuil a Maurepas, 29 octobre 1724, AC, C 11A, 46: 106-115. Par exemple: "Les esmolument de ce gouvernement ne valle pas un escu quoi que les a pointement soit considerable... Ce pays est a presan un pays de boeme il ni a pas de iour quon ne vaule a dix an droie au moins dans cette ville.

38. [ Adèle Cimon ], Les Ursulines de Québec depuis leur établissement jusqu'à nos jours (4 vol., Québec 1863-1866), 1: 484.

39. Coll. de Mss., 1: 582-589; Roy (24-27) reproduit un extrait de la "Relation de ce qui s'est passé de plus considérable en Canada depuis le départ de la frégate La Fleur de May, le 27 novembre 1690, jüsqu'au départ de 1691"; Frontenac à Pontchartrain, 20 octobre 1691, RAPQ (1927-1928), 68.

40. Frontenac à Pontchartrain, 15 septembre 1692, ibid., $115 \mathrm{~s}$.

41. Frontenac à Louis XIV, 25 octobre 1696, RAPQ (1928-1929), 307s. 
l'année suivante avec une augmentation d'appointements ${ }^{42}$. Il se rend de nouveau dans le vieux pays en 1694 , voit le ministre, auprès de qui il soutient les officiers réformés et revient, en 1695, avec une commission de capitaine de vaisseau ${ }^{43}$. En 1696, un procès avec sa belle-sœur nécessiterait sa présence dans la métropole, mais, n'osant solliciter tout de suite un troisième congé, il confie ses intérêts à sa femme ${ }^{44}$. Comme celle-ci ne parvient pas à faire régler cette contestation qui est pourtant "de la dernière conséquence", le commandant des troupes s'embarque à l'automne de $1697^{45}$; il ne perd pas son temps: on le voit à Paris, où ses petites industries lui valent le désagrément d'une réprimande ${ }^{46}$, on le voit surtout à la Cour, où il est décoré de la croix de Saint-Louis et se fait commettre pour recevoir chevaliers, à son retour, Frontenac, Callière et le marquis de Crisa$\mathrm{fy}^{47}$. Vaudreuil jouit maintenant d'un grand crédit aussi bien en France qu'au Canada. Il n'a pas été malaisé de s'en rendre compte en 1697. Cette année-là, Louis XIV a confié au marquis de Nesmond une puissante escadre avec la mission de consolider les gains réalisés par Iberville à Terre-Neuve, de couvrir l'entrée du Saint-Laurent, de s'emparer de Boston, de piller les côtes de la Nouvelle-Angleterre et - "vous me rendriez un service très agréable", ajoute le roi - d'attaquer New-York. Nesmond ne peut atteindre ses objectifs qu'en liaison avec un corps expéditionnaire canadien. A qui le souverain désire-t-il donner le commandement de ces troupes? A Frontenac, ou encore, si le vieux gouverneur ne peut être de la partie, à Vaudreuil.

42. Mémoire du Roy à Frontenac et à Champigny, [ 1692 ], RAPQ (1927-1928), 93; Mémoire du Roy à Frontenac et à Champigny, [ 1693 ], ibid., 142.

43. Frontenac et Champigny à Pontchartrain, 9 novembre 1694, RAPQ (19271928), 209; Mémoire du Roy à Frontenac et à Champigny, 14 juin 1695, RAPQ (1928-1929), 261; Frontenac a Pontchartrain, 25 octobre 1696, ibid., 315; Roy, 31.

44. Frontenac à Pontchartrain, 25 actobre 1696, RAPQ (1928-1929), 318; Hammang, 49s.

45. Frontenac et Champigny à Pontchartrain, 25 octobre 1698, ibid., 382. Par distraction, M. Pierre-Georges Roy affirme qu'en 1714 Vau'dreutil n'était pas allé en France depuis 1692, La Ville de Québec sous le régime français, 2: 24.

46. Pontchartrain à Vaudreuil, 12 mars 1698, AC, B 20: 15-15v.

47. Pontchartrain à Vaưdreuil, 19 juin 1698, ibid., 121; Pontchartrain à Bégon, 19 juin 1698, ibid., 101v-102. Voir Aegidius Fauteux, Les Chevaliers de Saint-Louis en Canada (Montréal, 1940), 50s, 91-94. 
Sa Majesté fait grand cas de l'expérience du chevalier ${ }^{48}$. De plus, on l'estime. Saint-Castin le déclarera bientôt: "Il est homme de guerre et n'est pas prévenu de lui-même, et capable d'écouter les ordres qu'on lui donnera. Joint à ce qu'il a beaucoup de douceur dans sa manière de commander et est aimé. On ira plus volontiers sous ses ordres que sous ceux d'un autre. ${ }^{49}$ " Un concurrent peut, à l'occasion, s'offusquer de son succès ${ }^{50}$, son bonheur s'explique.

Frontenac meurt le 28 novembre $1698^{51}$. Si le gouverneur de Montréal devient automatiquement administrateur intérimaire, rien ne s'oppose à ce que le roi nomme un autre que lui au gouvernement général. Vaudreuil le sait, il croit son heure venue, il brigue la nomination. Sa bonne étoile veut qu'il soit justement à Québec, où son quatrième fils vient de naître. Comme bien d'autres, Callière n'est pas sans connaitre l'ambition du commandant des troupes. Prévenu très tôt, il s'enferme dans une secrète activité. Il fait des lettres et les confie au sieur de Courtemanche, qui prend en toute hâte le chemin de la capitale; mais, arrivé à Sorel, le messager profite de la nuit pour disparaître: il oblique vers Albany; on l'attend encore à Québec, que le voilà déjà en route pour la France, par la voie de New-York. Le temps passe. Vaudreuil et Champigny s'avisent enfin qu'on leur joue "un tour de Normand". Vite, ils remettent leurs dépêches au sieur de Vincelotte et l'envoient en NouvelleAngleterre, où il s'embarquera, lui aussi, à destination de la métropole. L'intendant appuie de toutes ses forces la candidature du commandant des troupes. Il ne veut pas de Callière, Callière est un malade et, fait-il entendre, le service se dispense facilement de lui: “J'ay... apris... qu'il etoit fort incommodé, cela ne m'a pas surpris, car il l'est presque toujours, ce qui ne doit pas neantmoins vous em-

48. "Instruction à monsieur le marquis de Nesmond," 21 avril 1697, Coll. de Mss., 2: 263-268; Pontchartrain à Frontenac, 6 mars 1697, RAPQ (1928-1929), 325s; Louis XIV à Frontenac, 21 àvil 1697, ibid., 327s; Mémoire du Roy à Frontenac et à Champigny, 27 avril 1697, ibid., 330. Sur les causes de l'échec de Nesmond, qui ne put même pas se mettre en campagne, voir Frontenac à Pontchartrain, 15 octobre 1697, ibid., 339; Pontchartrain à Frontenac, 21 mai 1698, ibid., 360.

49. "Mémoire sur l'expédition contre Baston par monsieur de Saint-Castin," 1702, Coll. de Mss., 2: 398.

50. Frontenac a Pontchartrain, 10 octobre 1698, AC, C 11A, 16: 47-48.

51. Champigny à Pontchartrain, 22 décembre 1698, AC, C 11A, 16: 155. 
barrasser, les affaires du Roy n'en souffriront pas ${ }^{52}$ ". Qui des deux rivaux l'emportera? L'hiver et le printemps s'écoulent dans l'incertitude. Les Canadiens politiquent avec délices. Vaudreuil a sa cour et Callière la sienne. A la fin de mai, lorsque ce dernier décide de passer les troupes en revue, un incident éclate: n'exige-t-il pas que les officiers le saluent de la pique! Distinction réservée à un maréchal de France, déclare le contrôleur Le Roy de La Potherie, qui donne lecture des règlements et ordonnances du roi. Vaudreuil goûte fort les raisons du fonctionnaire et les soutient tant qu'il peut. Les ordonnances, tranche Callière, ne valent que pour la Marine, et nous ne sommes pas de la Marine. Dans sa version de la dispute, Vaudreuil se montre scandalisé: "Je demande, Monseigneur, si nous avons d'autre ministre que vostre grandeur... Pour moy, Monseigneur, je ne reconnois que vostre grandeur"53. Flatterie perdue: le 20 avril 1699, sur la recommandation de son frère, qui a travaillé à la glorieuse paix de Ryswick, Callière a obtenu le poste de gouverneur général ${ }^{54}$. Vaudreuil doit se contenter du gouvernement de Montréal ${ }^{55}$.

Un semblable épisode ne s'oublie pas. A Callière, le secrétaire d'État doit recommander de ne pas tenir rigueur de leur attitude à ceux qui ne sont pas entrés dans ses intérêts ${ }^{56}$. A Vaudreuil, il conseille d'oublier les "préventions" qu'il entretient contre un rival heureux; il ajoute: "J'ay parlé à $\mathrm{Sa} \mathrm{Ma}^{\mathrm{t} t}$ de la demande que vous faites de la lieutenance generalle de la Nouvelle France, mais elle n'a pas jugé a propos de la créer" ${ }^{\prime 57}$. Le chevalier se console en observant la

52. Ibid., 156. $157-161$.

53. Vaudreuil et La Potherie à Pontchartrain, 31 mai 1699, AC, C 11A, 17:

54. "Provisions de Gouverneur et Lieutenant General en Canada pour M. le Cher de Callieres," 20 avril 1699, AC, B 20: 168-168v. Gédéon de Catalogne narre avec humour les épisodes qui ont marqué la rivalité de Callière et de Vaudreuil, Coll. de Mss., 1: 601-603. Cf. Hammang, 50-53. Sur la nomination de Callière, voir aussi la sœur Françoise Juchereau de Saint-Ignace, Histoire de l'Hotel-Dieu de Québec (Montauban, [1751 ]), 379. A propos de François de Callière, consulter "Le Frère de notre gouverneur de Callières," BRH, 33 (1927): 48-51.

55. "Provisions de Gouverneur de l'Isle de Montreal dans la Nouvelle France pour le Sr de Vaudreuil Commandant des troupes," 28 mai 1699, AC, B 20: 224-225: Pontchartrain à Champigny, 30 mai 1699, ibid., 232.

56. Pontchartrain à Callière, 27 mai 1699, ibid., 200v-201v.

57. Pontchartrain à Vaudreuil, 31 mai 1701, AC, B 22: 210v-213. 
mauvaise santé de son chef $^{58}$. Il se fait construire à Montréal une belle maison, il soutient avec honneur son emploi ${ }^{59}$, il devient grand propriétaire $^{60}$, son revenu est bon $^{61}$. Pratique, madame de Vaudreuil fait sa cour au ministre ${ }^{62}$. Callière s'use lentement. Il meurt le 26 mai 1703 . Un mois plus tard, des funérailles solennelles ont lieu à Québec. Le supérieur des Récollets, le Père Gelase, prononce l'oraison funèbre: "Quand je considère tous les états de ce pays dans la consternation et dans les larmes, je vous avoue, Messieurs, que mon cœur, pénétré de la plus vive douleur, s'abandonnerait plus volontiers aux siennes qu'il ne permettrait à ma langue d'en exposer le sujet." Mais sa langue se délie; s'il pense au mort, l'orateur n'oublie pas les vivants; il se garde bien d'oublier Vaudreuil: "Notre douleur serait sans remède si la présence et l'espérance d'un chef en qui nous voyons briller la sagesse de Salomon, la bonté de Tite et la valeur des Césars ne rendait à nos cœurs la lumière par la connaissance qu'a tout le pays de sa rare prudence, le repos à nos esprits par l'expérience qu'on a depuis longtemps de son courage et à nos cœurs la consolation et la joie par tous les traits de bonté et d'affabilité, de générosité, de bienveillance, de clémence et de douceur propres à perpétuer les vraies délices des hommes dans cette NouvelleFrance" ${ }^{\prime 63}$.

Le pays ne semble pas douter que le chevalier ne recueille la succession de Callière: on assure que le peuple le désire ${ }^{64}$ et que le

58. Vaudreuil à Pontchartrain, 4 novembre 1702, AC, C 11A, 20: 145. Voir Françoise Juchereau, Histoire de l'Hótel-Dieu de Quebec, 409.

59. Champigny a Pontchartrain, 7 novembre 1701, AC, C 11A, 19: 140.

60. L'acte de concession de la seigneurie de Vaudreuil est du 12 octobre 1712; sur ce fief voir P.-G. Roy, éd., Inventaire des concessions en fief et seigneurie, fois et hommages et aveux et denombrements conservés aux archives de la province de Québec (6 vol., Beaùceville, 1927-1929), 4: 170. En 1721, le seigneur y aura distribué vingt concessions: voir I. Caron, éd., "Procès-verbaux sur la commodité et incommodité dressés dans chacune des paroisses de la Nouvelle-France," RAPQ (1921-1922), 304.

61. Voir Hammang, 45, note 16.

62. Pontchartrain à madame de Vaudreuil, 31 mai 1701, AC, B 22: 213v.

63. "Eloge funèbre de feu messire Hector de Callières," 26 juin 1703, RAPQ (1921-1922), 228, 231. La mort de Callière permet immédiatement à Vaudreuil de prendre le titre de commandant général, Vaudreuil et Beauharnais à Pontchartrain, 15 novembre 1703, Coll. de Mss., 2: 405.

64. Les Ursulines de Quebec, 2: 138; P.-F.-X. de Charlevoix, Histoire et description générale de la Nouvelle-France, avec le Journal Historique d'un Voyage fait par ordre du Roi dans l'Amerique Septeritrionale (3 vol., Paris, 1744), 2: 288. 
clergé le demande ${ }^{65}$. Aucune concurrence ne se laisse entrevoir, et la protection de Pontchartrain est toute-puissante. Le ler aout 1703, Louis XIV nomme Philippe de Rigaud gouverneur général et lui donne le titre de marquis de Vaudreuil ${ }^{66}$. Une circonstance a cependant failli coûter à ce dernier la promotion: il a épousé une Canadienne qui a des parents dans la colonie; le ministre lui révélera n'avoir pu vaincre les répugnances du roi "que sur les asseurances que je luy ay donné... que vous agiriez avec les parens de votre femme comme s'ils ne l'estoient pas'"67. Très tôt, les adversaires du marquis agitent comme un épouvantail la parenté de la marquise. Le gouverneur s'en plaint dès $1704^{68}$. En 1705, Ruette d'Auteuil l'accuse de se laisser "gouverner" par le premier conseiller Chartier de Lotbinière, oncle de madame de Vaudreuil ${ }^{69}$. De pareilles dénonciations impressionnent Pontchartrain. Il a été heureux, confie-t-il un jour à la marquise, de faire donner à son mari le gouvernement général, mais, poursuit-il, "pour l'amour de luy et de vous mesme vous devez l'engager a ne pas traitter les gens qui ont l'honneur de vous apartenir dans ce pays d'une autre maniere que les autres habitans de la colonie" $^{\prime 70}$.

Lorsque Vaudreuil prend en mains les destinées de la NouvelleFrance, le pays s'engage dans une longue période de crise et d'insé-

65. "Lorsque l'état ecclésiastique vous a écrit en sa faveur pour vous demander, Monseigneur, votre puissante protection pour lui faire obtenir de S.M. le gouvernement général de ce pays, ils ne croyaient pas qu'il en fit un si mauvais usage," Ramezay a Pontchartrain, 14 novembre 1704, dans Ivanhoë Caron, "Inventaire de dơcuments concernant l'Eglise du Canada sous le régime francais," RAPQ (19401941), 377. Voir la version de la sœur Françoise Juchereau, Histoire de l'Hótel-Dieu de Québec, 420.

66. "Provisions de Gouverneur et Lieutenant-Général en Canada, Acadie, Isle de Terre-Neuve et autres pays de la France Septentrionale pour Monsieur le Marquis de Vaudreuil." Ces provisions sont enregistrées au greffe du Conseil souverain, à Québec, le 17 septembre 1705. Edits et ordonnances (3 vol., Québec, 1854-1856), 3: 58s. Gagnion affirme à tort que Vaudreuil a porté le titre de marquis "depuis la mort de son père, tứ à la bataille de Luzzara, en 1702", Le Fort et le chateau Saint-Louis, 60. Le titre est honoraire, Hammang, 41.

67. Pontchartrain à Vaudreuil, 9 juin 1706, AC, B 27: 225.

68. Vaudreuil a Pontchartrain, 16 novembre 1704, AC, C 11A, 22: 65.

69. Ruette d'Auteuil à Pontchartrain, 17 octobre 1705, RAPQ (1922-1923), 16; Pontchartrain à Lơtbinière, 9 juin 1706, AC, B 27: 277v-278.

70. Pontchartrain a Mme de Vaudreuil, 9 juin 1706, AC, B 27: 271-271v; id. à $i d ., 30$ juin 1707, RAPQ (1939-1940), 377. 
curité. La France est en guerre, et la guerre tourne mal. Cependant la politique du marquis ne manque ni d'habileté ni de cohérence; il l'a exposée en arrivant au pouvoir: ne rien entreprendre contre le NewYork afin de conserver la neutralité des Iroquois, mais organiser des raids, "brouiller les cartes" du côté de la Nouvelle-Angleterre pour empêcher les Abénakis de devenir des neutres ${ }^{71}$. Le ministre aura mauvaise grâce de lui reprocher son "inaction" "72, puisque le gouverneur réussit, au Canada, à sauver presque toute la mise, alors que la métropole perd la partie. Et qu'est-ce que le Canada? Un pays tout petit par sa population, - 15,000 âmes en 1698 et 18,000 en 1713 , - mal guéri des blessures que lui ont infligées, avant 1700 , les hostilités iroquoises, dépouillé par la paix d'Utrecht du territoire de l'Acadie, des pêcheries de Terre-Neuve et des fourrures de la baie d'Hudson et, de surcroît, ébranlé dans la structure même de son économie. L'inflation détraque les finances coloniales pendant que les pertes maritimes engloutissent des fortunes. Et il y a la déplorable affaire de la Compagnie de la Colonie, œuvre de la Cour et de l'aristocratie de la pelleterie; formée en 1700 , elle s'effondre en 1706 , grevée d'une dette de près de deux millions. Les temps sont durs ${ }^{73}$. Malgré tout, malgré les craintes les plus vives et les pires déboires, Vaudreuil parviendra très tôt à faire oublier ces années sombres. Après sa mort, on écrit: "C'est avec justice que nous l'avons pleuré, car sous sa vigilante administration, le Canada a joui d'une prospérité jusqu'alors inconnue. Pendant vingt-deux ans, le cultivateur, le commerçant et le militaire n'ont eu également qu'à bénir son nom" Trente ans plus tard, on évoque encore les "jours fortunés" de son gouvernement ${ }^{75}$. Se faire aimer et respecter ${ }^{76}$ dans des conjonctures aussi difficiles exige plus que du talent: le gouverneur a dû y mettre une continuelle application.

71. Vaudreuil à Pontchartrain, 14 novembre 1703, AC, C 11A, 21: 84; id à $i d$., 16 novembre 1704, ibid., 22: 51.

72. Pontchartrain à Vaudreuil, 6 juin 1708, Coll. de Mss., 2: 489. 26-29.

73. Voir G. Frégault, La Civilisation de la Nouvelle-France (Montréal, 1944),

74. Les Ursulines de Québec depuis leur établissement jusqu'à nos jours, 2: 140.

75. [ Courville], Mémoires sur le Canada, depuis 1749 jusqu'à 1760 (Québec, 1873), 58.

76. Hocquart à Maurepas, 30 septembre 1733, AC, C 11A, 60: 19. 
Pendant ce temps, aux soucis de l'État, s'ajoutent des préoccupations personnelles. Ce n'est pas tout que d'arriver, il faut encore se maintenir. Le gouverneur doit compter avec des adversaires peu nombreux, mais dangereux, qui se recrutent surtout parmi les directeurs de la Compagnie de la Colonie et parmi les traitants déguisés en fonctionnaires: Ruette d'Auteuil, Ramezay, Cadillac. Cadillac, bien que "haï de tous les Français et sauvages" "77, se croit autorisé à lancer les accusations les plus fantastiques ${ }^{78}$. Dès 1703, Vaudreuil s'est attiré les foudres de l'ambitieux trafiquant en disant la vérité sur l'entreprise commerciale de Détroit ${ }^{79}$. Le gouverneur de Montréal, Ramezay, qui a la fatuité ombrageuse comme d'autres ont le vin triste, s'en prend au marquis avec violence; il lui reproche de livrer la colonie aux Jésuites ${ }^{80}$. De son côté, Vaudreuil assure que Ramezay cherche à le brouiller avec l'intendant, qu'il répond aux gens à coups de bâton, - “ces manieres font trembler tout le monde", et qu'il vaudrait mieux qu'il ne commandât point en l'absence du gouverneur général ${ }^{81}$. Par solidarité avec Ramezay, Daigremont insinue que le marquis extorque de gros présents aux sauvages ${ }^{82}$. Ruette d'Auteuil va plus loin: Vaudreuil, déclare-t-il, pratique la traite de l'eau-de-vie ${ }^{83}$.

Ces personnages ont pourtant moins de poids qu'un intendant, haut fonctionnaire presque toujours tenté de se poser en rival du gouverneur. Avec Beauharnais, apparenté au comte de Pontchartrain, le marquis passe deux années sans histoire. Les successeurs de Beauharnais, Jacques Raudot et son fils, Antoine-Denis Raudot,

77. Daigremont à Pontchartrain, 14 novembre 1708, RAPQ (1940-1941), 413. 20,38 .

78. Voir Jean Delanglez, “Cadillac's Last Years," Mid-America, 33 (1951):

79. Vaudreuil à Pontchartrain, 14 novembre 1703, AC, C 11A, 21: 86; id. à id., 16 novembre 1704, ibid., 22: 55-56; Vaudreuil à Pontchartrain, 14 novembre 1709, RAPQ (1942-1943), 439.

80. Ramezay à Pontchartrain, 14 novembre 1704, RAPQ (1940-1941), 376-378.

81. Vaudreuil à Pontchartrain, 16 novembre 1704, AC, C 11A, 22: 62-63.

82. Daigremont à Pontchartrain, 14 novembre 1708, RAPQ (1940-1941), 413s; Pontchartrain à Vaudreuil, 6 juillet 1709, RAPQ (194i-1943), 406; Vaudreuil a Pontchartiain, 14 novembre 1709, ibid., 438. 16-17.

83. Ruette d'Auteuil à Pontchartrain, 17 octobre 1705, RAPQ (1922-1923), 
tiennent également à la famille du ministre. Ils arrivent à temps pour assister, en 1705, à la réception officielle de Vaudreuil, cérémonie qui se déroule "avec beaucoup de pompe $\&$ un grand concours de peuple" ${ }^{\prime 84}$. Jacques Raudot est un vieux lettré qui discourt admirablement de toutes choses; amateur de musique, rien ne le délasse de ses graves travaux comme d'écouter " un concert mêlé de voix, \& d'instrumens". Son fils, jeune homme très sage, a, rapporte-t-on, "quelque chose des anciens Philosophes Stoïciens" ${ }^{85}$. Auprès d'eux, le marquis apparaît comme un homme sans culture; à sa mort, on ne trouvera chez lui que quelques livres: une Nouvelle description de la France par le sieur Pignariol de Lafore, une Géographie historique ou description de l'univers, les Epîtres et évangiles pour toute l'année, en deux tomes, les Voyages et aventures de Franois Le Gate, aussi en deux tomes, et la Vie et aventures de Robinson Crusoé, toujours en deux tomes $^{86}$. Durant trois ou quatre ans, le militaire et le magistrat s'entendent toutefois à merveille. En 1709, tout change. Jacques Raudot estime-t-il soudain son collègue trop semblable à ces grossiers Canadiens qu'il entend corriger "de la violence dans laquelle [ la ] férocité de leurs mours les jette souvent, en les condamnant à de bonnes amendes" ${ }^{\prime 87}$ ? On ne sait. Il aveint son écritoire et compose une longue épître. Il s'évertue à expliquer le silence complice qu'il observe depuis des années. Il en demande pardon: il n'a pas osé faire "de la peine" au ministre en dénonçant quelqu'un que celui-ci protège. Puis il donne libre cours à sa vertueuse indignation. Les pages s'empilent, noires d'accusations: Vaudreuil est un "despote" qui terrorise la colonie, un "ignorant" gonflé "d'opiniastrete" qui tient le peuple dans l'oppression, "persuadé qu'il peut dire: je veüx et qu'à ce mot tout doit fléchir"; la Nouvelle-France tremble: "On peut dire avec raison que ce pays-cy est plus malheureux que si l'inquisition y estoit etablie"; trois passions dominent le gouverneur et la "Gouvernante": "l'esprit d'interet, de vengeance et d'avarice"; la femme et

84. Françoise Juchereau, Histoire de l'Hôtel-Dieu de Québec, 420s.

85. Ibid., 463.

86. "Inventaire et description" des biens de Vaudreuil, 19-25 juin 1726, RAPQ (1921-1922), 248. 406.

87. Jacques Raudot a Pontchartrain, 10 novembre 1707, RAPQ (1940-1941), 
le mari "ne sont occupez... que de l'envie d'amasser du bien et mesme ils ne s'en cachent pas, prenant pour prétexte qu'ils ont une grosse famille". A quoi attribuer cette philippique? Raudot taxe quelque part le marquis d'avilir les magistrats, contre qui il laisse circuler des coupletš irrévérencieux. "On m'a aussy chanté", déclare l'intendant, qui aime pourtant la musique vocale. Bien plus, la marquise elle-même a osé faire sur lui une chanson badine. On a beau dire, à Paris, tout ne finit pas par des chansons. Pontchartrain, qui a pourtant dû rire sous cape, envoie la dépêche à l'ancien intendant et au premier commis de la Marine avec cet avis: "M. de Beauharnais lire tout cela Et me rendre cette lettre Il faudra que nous en parlions/ M. de la Touche à bien examiner - important dans le temps qu'on travaillera a toutes ces affaires-la' ${ }^{\prime 88}$. En 1710, le secrétaire d'État gourmande le gouverneur en des termes où, c'est clair, il reste quelque chose des vitupérations de Raudot, mais sur un ton qui donne à penser que l'on n'a pas pris au tragique les éloquents discours du vieil intendant ${ }^{89}$. Le plus piquant de toute l'aventure est que, plus tard, Riverin représentera les deux Raudot sous les traits d'aventuriers "nez sans biens... [ et ] rapellés sur les plaintes generales qui ont eté faittes par tous les peuples de cette colonie, aprés les avoir tous vexé, s'etre enrichi, et avoir fait chasser et deserté de ce pays presque tous les principaux qui ont voulu leur resister" ${ }^{\prime 90}$. Ruette d'Auteuil mettra dans le même sac intendant et gouverneur, ces deux "instruments d'iniquite'"91.

Que va faire Vaudreuil? Attendre, laisser passer l'orage? Rien ne serait plus mal avisé. Ce n'est pas une coincidence qu'à l'automne de 1709 , même si le roi n'a pas envoyé son vaisseau annuel, madame de Vaudreuil se sente si pressée d'aller en France qu'elle s'embarque sur un petit navire marchand, malgré les risques et l'incommodité d'une traversée effectuée dans de pareilles conditions; risques trop $228 v$.

88. Jacques Raưdot à Pontchartrain, 20 septembre 1709, AC, C 11A, 30: 146-

89. Pontchartrain à Vaudreuil, 10 mai 1710, RAPQ (1946-1947), 372s; Mémoire du Roy a Vaudreuil et à Raudot, 10 mai 1710, ibid., 374. Raudot avait d'ailleurs commencé à se plaindre l'année précédente: voir Pontchartrain à Vaudreuil, 6 juillet 1709, RAPQ (1942-1943), 406.

90. Riverin au comte de Toulouse, 9 avril 1716, AC, C 11A, 36: 381.

91. "Mémoire sur l'état présent du Canada," 1712, RAPQ (1922-1923), 50. 
réels, puisque la Bellone, à bord de laquelle elle voyage, se fait prendre par un vaisseau britannique dont le capitaine, honnête homme a la vérité, montre de la considération pour la marquise, qu'il débarque proche le Havre ${ }^{92}$. Madame de Vaudreuil, on le sait aujourd'hui, ne passe pas dans la métropole parce que la Cour, où on ne la connait encore que très peu, lui aurait mandé en 1708 de venir occuper le poste de sous-gouvernante des "enfants de France"93, c'est-à-dire de la famille du duc de Berry. Elle ne se verra offrir cette charge qu'en 1712, et le duc d'Alençon, destiné à ses soins avant de naître, ne vivra que trois semaines; le duc de Berry, au milieu de 1713, ne la retient pas moins "pour l'éducation des autres enfants qu'il aura par la suite", mais il n'en aura qu'un, mort presque aussitôt que né. Et la marquise reste quand même à la Cour. Elle s'y emploie à soutenir son mari et à pousser ses fils. Dès son arrivée en France, elle explique, elle défend la politique du marquis, elle insiste sur la modicité - relative - des appointements du gouverneur et elle demande des "grâces": une pension pour Vaudreuil, une promotion pour un de ses fils, un bénéfice pour un autre ${ }^{94}$. Jusqu'ici, le marquis a maintes fois sollicité en faveur de sa famille, "qui augmente tous les jours"; éloigné du ministre, il lui a le plus souvent fallu se satisfaire de peu ${ }^{95}$. Plus heureuse, madame de Vaudreuil obtient tout de suite beaucoup ${ }^{96}$. C'est qu'elle déploie une étonnante activité. En 1710, elle présente a Pontchartrain un mémoire où il est longuement question de politique, de grande et de petite politique: elle s'y entend; elle n'a pas

92. Françoise Juchereau, Histoire de l'Hôtel-Dieu de Québec, 455.

93. Contrairement à l'opinion exprimée à plusieurs reprises, mais sans preuves, par M. Pierre-Georges Roy, La Ville de Québec sous le régime français, 2: 25s; Les Petites Choses de notre histoire, 4: 193; La Famille de Rigaud de Vaudreuil, 42. Ce n'est que le 4 juillet 1713 que Pontchartrain annonce a Vaudreuil que la femme de ce dernier a été nommée sous-gouvernante et que le duc de Berry retient ses services pour l'avenir, AC, B 35: 109v. Voir aussi Hammang, 64.

94. Résumé d'une lettre de Mme de Vaudreuil à Pontchartrain, 1709, RAPQ (1942-1943), 414-417.

95. Vaudreuil à Pontchartrain, 16 novembre 1704, AC, C 11A, 22: 64-65; Beauharnais à Pontchartrain, 17 novembre 1704, ibid., 70; Vaudreuil à Pontchartrain, 16 octobre 1705, RAPQ (1938-1939), 74; Pontchartrain d Vaudreuil, 9 juin 1706, AC, B 27: 229v-230; id. à id., 6 juin 1708, AC, B 29: 350.

96. Pontchartrain a Vaudreuil, 10 mai 1710, RAPQ (1946-1947), 373; Vaudreuil à Pontchartrain, 25 octobre 1710, ibid., 386; id. à $i d ., 3$ novembre 1710, ibid., 403. 
perdu son temps, puisque le ministre lui a déjà promis de lui faire remettre un mémoire des plaintes que l'on porte contre Vaudreuil ${ }^{97}$. Prévenu, celui-ci pourra mieux parer les coups d'adversaires qu'il reconnaîtra.

Il est sûr, écrit en 1712 Ruette d'Auteuil, que l'attitude du secrétaire d'État à la Marine "encourage Mme de Vaudreuil à mettre tout en usage pour hausser et étendre l'opinion de la protection de ce ministre dont elle se vante, il est certain qu'il lui en accorde une bien éclatante; il ne lui refuse rien, elle dispose de tous les emplois du Canada, elle écrit de toutes parts dans les ports de mer des lettres magnifiques du bien et du mal qu'elle peut faire auprès de lui, elle off re sa faveur, elle menace de son crédit;... Mr de Pontchartrain de son côté fait tout ce qu'il faut pour l'autoriser et justifier ses discours" qu'une femme qui regne tant presente qu'absente" ${ }^{\prime 99}$. Le marquis éprouve les effets de la faveur que sa femme s'est acquise. En 1712, il est gratifié d'à peu près tout ce qu'il veut ${ }^{1}$. Quand le roi le nomme commandeur de l'ordre de Saint-Louis, madame de Vaudreuil en est prévenue la première ${ }^{2}$. Sans hésiter, celle-ci sollicite immédiatement une gratification pour le gouverneur. Le gouverneur, répond le ministre, vient de recevoir une faveur insigne; elle "doit bien vous contenter l'un et l'autre"'3. Contente, la marquise l'est sans doute, mais contentée, pas tout de suite. Elle désire des croix de Saint-Louis pour quelques-uns de ses protégés, un brevet pour un autre, plus de pouvoir et plus de fret pour Vaudreuil, pour Vaudreuil encore la ratification d'une concession où la traite se pratique plus que le dé-

97. RAPQ (1946-1947), 406-410. La même année, elle discute d'un projet d'invasion de l'Acadie, Coll. de Mss., 2: 513.

98. Ruette d'Auteuil, "Mémoire sur l'état présent du Canada," 1912, RAPQ (1922-1923), 50.

99. Riverin au comte de Toulouse, 9 avril 1716, AC, C 11A, 36: 384. Au sujet de Riverin, voir les nombreux documents reproduits dans "Notes sur Denis Riverin," BRH, 34 (1928): 65-76, 129-139, 193-206.

1. RAPQ (1946-1947), 446s.

2. "N'en parlez point je vous prie," Pontchartrain à Mme de Vaudreuil, 18 juin 1712, AC, B 34: 25v.

3. Pontchartrain à Mme de Vaudreuil, 8 juillet 1712, AC, B 34: 96v-97. 
frichage $e^{4}$ Elle veut enfin que son fils aîné soit promu lieutenant de vaisseau. "Je vous avoue que votre demande me paroist fort prematurée", déclare cette fois Pontchartrain, qui ajoute: "Il faut de la moderation en tout parceque $\mathrm{Sa} \mathrm{Ma}^{\text {té }}$ ne pouroit pas trouver bon les demandes trop frequentes"...5 Réponse tout à fait raisonnable. Six mois plus tard, le fils Vaudreuil n'en devient pas moins lieutenant de vaisseau ${ }^{6}$. Il a 22 ans. L'année suivante, sa mère voudrait le voir major des troupes, mais le roi le juge trop jeune ${ }^{7}$.

La guerre s'achève, et le marquis songe à revoir sa femme et son pays. En 1714, le secrétaire d'État lui procure l'agrément d'aller "faire un tour" en France ${ }^{8}$. Jamais voyage ne saurait tomber plus à propos. Un nouvel intendant, Michel Bégon, est arrivé à l'automne de 1712. Issu d'une famille distinguée de fonctionnaires maritimes', fils d'un intendant de Rochefort et de la Rochelle allié à Colbert, le magistrat apporte à Québec les goûts magnifiques de son père, collectionneur averti et esprit raffiné, la passion du négoce, qui lui vaudra au bout du compte moins de profits que d'embarras financiers, et la volonté de tenir tête au gouverneur, contre qui Raudot l'aurait prévénu ${ }^{10}$. Écrivent-ils en commun, les deux administrateurs protestent, il est vrai, de leur "envie de vivre dans une parfaite intelligence"11. Ils restent quand même sur leurs positions. Dans la métropole, Vaudreuil saisit l'occasion de rétablir la situation. Ce n'est pas par hasard, semble-t-il, qu'à l'été de 1715 Pontchartrain adresse

4. Pontchartrain à Mme de Vaudreuil, 27 avril 1712, AC, B 34: 2; Pontchartrain à Bégon, 25 juin 1712, ibid., 61v., 66v; Pontchartrain à Mme de Vaudreuil, 9 juillet 1712, ibid., 98v; "Demandes du Canada," 1712, AC, C 11A, 33: 443.

5. Pontchartrain à Mme de Vaudreuil, 18 décembre 1712, AC, B 34: 147-147v.

6. Pontchartrain à Vaudreuil, 4 juillet 1713, AC, B 35: 109-109v; Pontchartrain à Louis-Philippe de Vaudreuil, 4 juillet 1713, ibid., 110-110v.

7. "Remplacement des employés militaires," 7 mai 1714, AC, C 11A, 34: 506; Pontchartrain à Vaudreuli, 24 mai 1714, AC, B 36: 411.

8. Pontchartrain à Vaudreuil, 19 mars 1714, AC, B 36: 355.

9. Yvonne Bezard, Fonctionnaires maritimes et coloniaux sous Louis XIV: les Bégon (Paris, 1932).

10. Hammang, 75. 374.

11. Vaudreuil et Bégon à Pontchartrain, 12 novembre 1712, BRH, 33 (1927): 
de vifs reproches à Bégon. Il lui est soudain revenu "par différents endroits" que l'intendant spécule sur le blé, réquisitionne les farines pour effectuer aux Antilles des exportations dont il prend le monopole, s'empare du commerce de la viande, réglemente à son seul avantage le cabotage sur le Saint-Laurent et, en un mot, aspire à se rendre maitre "de tout le commerce du Canada"12. Semonce méritée. Le magistrat aurait perdu 50,000 livres dans l'incendie de son palais en janvier $1713^{13}$ et, explique-t-on, il n'a de cesse qu'il s'en soit "dédommagé" sans rien rabattre de sa "générosite" ni rien retrancher de son fastueux train de vie ${ }^{14}$. Il publie des ordonnances qui confirment toutes les dénonciations et autorisent tous les soupçons ${ }^{15}$. Il s'avoue d'ailleurs coupable ${ }^{16}$. Coupable, le marquis estime aussi que son collègue l'a été envers le gouverneur par une attitude de constante opposition. Que peut-il en résulter, demande le ministre, sinon des brouilleries nuisibles au service? Il faut que M. de Vaudreuil "soit d'un bon esprit et d'un caractere bien doux pour que cela n'ait pas fait cet effet"17. Le marquis triomphe.

Sur les entrefaites, Louis XIV meurt, et Saint-Simon - c'est, par bonheur, un bienfaiteur de la marquise de Vaudreuil - obtient la tête de Pontchartrain. Les ministères disparaissent, remplacés par des conseils; les colonies relèveront désormais du Conseil de Marine ${ }^{18}$. Le gouverneur a la chance inouïe d'être près de la Cour: absent, ne risquerait-il pas d'avoir tort? D'autant que ses vieux adversaires relèvent la tête. Toujours éloquent et toujours passionné, Ruette

12. Pontchartrain à Bégon, 13 juillet 1715, AC, B 37: 204-205; Pontchartrain à l'abbé Bégon (frère de l'intendant), 17 juillet 1715, ibid., 134v-136v.

13. Vaudreuil a Pontchartrain, 11 février 1713, AC, C 11A, 34: 27-28; Francolse Juchereau, Histoire de l'Hôtel-Dieu de Québec, 498-502.

14. Monseignat au Conseil de Marine, 4 novembre 1717, AC, C 11A, 37: 397 399; Hocquart a Maurepas, 17 octobre 1730, AC, C 11A, 53: 83-84.

15. Voir les ordonnances des 24 janvier, 12 et 23 septembre, 27 octobre et 30 décembre 1714, 4 janvier, 26 février, 17 avril et 29 mai 1715 dans P.-G. Roy, éd., Inventaire des ordonnances des intendants de la Nouvelle-France conservees aux archives provinciales de Québec (4 vol., Beauceville, 1919), 1: 133-150, passim.

16. Le Conseil de Marine à Bégon, 16 juin 1716, AC, B 38: 210-211.

17. Pontchartrain à Bégon, 10 juillet 1715, AC, B 37: 172-174.

18. Le Conseil de Marine a Vaudreuil et Bégon, 3 novembre 1715, AC, B 38: 194-195v. 
l'accuse d'avoir gâté les relations de la Nouvelle-France et des Indiens à force de pratiquer la traite illégale ${ }^{19}$. Un autre revenant de la Compagnie de la Colonie, Denis Riverin, offre au Conseil des mémoires contre cet administrateur avide, "entierement gouverné par sa femme", persécuteur des Canadiens, qui en "gemissent journellement" 20 . Occupés à d'autres intrigues, les nouveaux maîtres n'ont que faire de ces récriminations. Bien plus, sentant son inexpérience, le Conseil invite même le gouverneur à prendre part à ses délibérations, au Louvre; on écoute ses avis, on les suit ${ }^{21}$. En février 1716, Vaudreuil présente de son côté un long mémoire, plein de faits et d'idées. Il y expose les besoins de la colonie et y définit son programme: "Profiter de la paix pour fortifier le Canada," ce qui veut dire: $\mathrm{y}$ envoyer des soldats et des colons, améliorer les pêcheries et le commerce des fourrures, fortifier le Cap-Breton, distribuer des présents aux sauvages pour empêcher ceux-ci de se jeter dans les bras des Anglais et accorder une aministie aux coureurs de bois ${ }^{22}$. Il y demande encore le rétablissement des congés et la permission d'établir chez les Indiens les postes qu'il jugera nécessaires; il obtient tout $^{23}$. Il désire enfin que sa voix soit prépondérante en cas de désaccord avec l'intendant; la Cour se rend à son vœu ${ }^{24}$.

Le gouverneur rentre à Québec plus puissant que jamais à l'automne de 1716. C'est maintenant un vieillard, la traversée lui a été pénible: en débarquant, il doit se faire "apporter" à l'Hôtel-Dieu, où il passe près d'un mois ${ }^{25}$. Il se remet à temps pour annoncer à la

19. "Mémoire sur les commerces de $\mathrm{M}$. de Vaudreuil avec les sauvages et les Anglais," RAPQ (1922-1923), 52; "Mémoire sur ce qui concerne le commerce des castors et ses dépendances," ibid., 71.

20. Riverin au comte de Toulouse, 9 avril 1716, AC, C 11A, 36: 381-385.

21. "Canada, Conseil," 5 mai 1716, AC, C 11A, 35: 379-386.

22. "Extrait du mémoire de M. de Vaudreuil, adressé à Son Altesse Royale Monseigneur le Duc d'Orléans, regent du Royaume," février 1716, copie aux Archives de la province de Québec.

23. Mémoire du Roy à Vaudreuil et à Bégon, 26 juin 1717, AC, B 39: 230, 231. Vaudreuil ne manque pas de beaux prétextes pour distribuer les congés à qui il veut, "Les Congés de traite accordés en 1717, BRH, 29 (1923): 271.

24. Mémoire du Roy à Vaudreuil et à Bégon, 15 juin 1716, AC, B 38: 221v. Vaudreuil obtient aussi, en mai 1716, que la Cour ratifie la concession de sa seigneurie, P.-G. Roy, éd., Inventaire des concessions en fief et seigneurie, 4: 171.

25. Françoise Juchereau, Histoire de l'Hôtel-Dieu de Québec, 550. 
Cour une victoire: le lieutenant de roi à Québec, Louis de Laporte de Louvigny, est allé battre les féroces Outagamis ou Renards, au fond de la baie des Puants [ Green Bay ]. D'un ton héroïque, il salue cette "premiere action de guerre qui se soit passée sous le Regne de Sa Majesté [ Louis XV], Glorieuse aux armes du Roy et tres utile pour cette Colonie" ${ }^{\prime 26}$. Il exagère. Louvigny est-il allé en traite ou en guerre ? Ses hommes ont transporté quantité de ballots de marchandises et quarante barriques d'eau-de-vie. Le commandant aurait pu écraser l'ennemi, il lui a donné la paix. Pourquoi ? Les Outagamis ont promis des castors ${ }^{27}$. Et la guerre reprend, elle durera encore vingt ans. Les Renards se regroupent et trouvent partout des alliés: chez les Sioux et les Iowa, chez les Maskoutins, les Kikapous et Puants [ Winnebago ]. Ils tombent sur les Illinois, massacrent des Français, occupent le portage de Chicago, infestent les voies de communication avec la Louisiane. Le gouverneur ferme les yeux. Sa complaisance est-elle intéressée ? On le dirait. En 1724, il envoie Le Marchand de Ligneris à la Baie. Ligneris bâcle une paix boîteuse qui sacrifie les Illinois aux Renards. Cette étrange politique fait scandale, on crie au désastre, la Cour s'émeut. Maurepas, à la Marine depuis 1723, fait une observation qui va loin: Vaudreuil ne serait pas pressé de mettre fin aux hostilités, dangereuses pour la Louisiane, mais profitables aux traitants du Canada. "On dit, écrit le ministre, que vous ne regardez pas les Français qui sont de la Louisiane comme Français, que vous ne vous souciés pas que les sauvages les regardent de même et qu'en faisant continuer cette guerre vous voulez empêcher les sauvages de porter leurs pelleteries du côté de cette nouvelle colonie et les faire tomber toutes en Canada" ${ }^{28}$. Les pelleteries, toujours les pelleteries. Pendant les dernières années de sa vie, le marquis en a l'obsession.

Tout d'abord, sa femme sollicite pour lui la permission d'établir un poste aux Témiscamingues avec "la propriété du commerce" qui s'y fera, excellent moyen, souligne-t-elle, de nous attacher les sau-

26. Vaudreuil au Conseil de Marine, 14 octobre 1716, AC, C 11A, 36: 77-78; voir Louvigny au Conseil de Marine, 14 octobre 1716, ibid., 159-162 et "Conseil, Guerre des Renards," 28 décembre 1716, ibid., 278-284. 146-148.

27. Clarence W. Alvord, The Illinois Country, 1673-1818 (Springfield, Ill., 1920),

28. Ibid., 160-162; F.-Emile Audet, Les Premiers Etablissements français au pays des Illinois. La guerre des Renards (Paris, 1938), 121-124. Voir Charlevoix, 2: 407. 
vages de la région et de les détourner de porter aux Anglais de la baie d'Hudson les plus belles fourrures du Canada. La Cour y consent. ${ }^{29}$ Aussitôt, les négociants se récrient: à les entendre, l'agent du gouverneur, Guillet, intercepte les convois à destination de Montréal plutôt que ceux qui se dirigent vers les comptoirs britanniques. Si Vaudreuil affecte de ne voir dans les protestations des marchands qu'une campagne de diffamation lancée par la famille d'Auteuil ${ }^{30}$, le ministre, conscient des tentations auxquelles succombent les fonctionnaires supérieurs de la colonie, craint que le vieux marquis ne s'immisce dans la traite et il lui pose nettement la question: "Que fait Guillet dans ce poste où il ne doit pas estre?" Le gouverneur éprouve un embarras visible à définir le rôle de son "fermier". Guillet, avoue-t-il, "fait publiquement le commerce", mais un commerce permis, prévu par la concession du poste de Témiscamingue. Voilà qui est "d'un mauvais exemple", riposte le secrétaire d'État; le roi n'a jamais accordé que le commerce "des denrées de la terre"; le marquis devra rappeler son agent, affermer régulièrement la traite de Témiscamingue, en appliquer les revenus aux fortifications de Montréal et, surtout, combattre la course des bois ${ }^{31}$. Sur un point de sa seigneurie de Vaudreuil, à l'île aux Tourtres, dans le lac des DeuxMontagnes, le gouverneur a un autre "fermier", François d'Youville, qui aime l'argent et plus encore les plaisirs que l'argent achète ${ }^{32}$. A force d'eau-de-vie, Youville dévalise les sauvages; la foire de Montréal s'en ressent. Tout le monde s'en plaint, sauf Vaudreuil, qui en retirerait plus de 10,000 écus ${ }^{33}$. Le gouverneur ne le nie pas, son agent s'adonne à la traite, mais, prétend-il, rien ne s'y oppose; une fois de plus, Maurepas ne goate point pareille opinion ${ }^{34}$. Il faut obéir,

29. Mme de Vaudreuil au comte de Toulouse, 1717, AC, C 11A, 38: 152-153.

30. Vaudreuil au Conseil de Marine, 20 octobre 1722, AC, C 11A, 44: 178.

31. Vaudreuil à Maurepas, 29 septembre 1723, AC, C 11A, 45: 86-92. Les commentaires du ministre en marge de cette dépêche sont du plưs haut intérêt.

32. Alber tine Ferland-Angers, Mèe d'Youville, première fondatrice canadienne (Montrésl, 1945), 35-38.

33. Ramezay a Maurepas, 15 octobre 1723, AC, C 11A, 45: 214-230. En marge le ministre note: "Ecrire a M. de Vaudreuil $p[$ ou ]r quil ait attention sur son fermier qui enivre les Sauvages."

34. Vaudreuil à Maurepas, 25 octobre 1724, AC, C 11A, 46: 66-67; Maurepas à Vaudreuil, 22 mai 1725, AC, B 48: 813. 
adjuger le poste de Témiscamingue. Bégon y procède dans les formes, au Palais, en présence du marquis. Après avoir suscitédes difficultés sur les limites de l'exploitation, celui-ci doit abandonner le privilège au plus haut enchérisseur, Fleury de La Gorgendière, quitte, sept mois après, à lui refuser la permission d'y envoyer du monde et des marchandises; si bien que Guillet trafiquera une année encore pour le compte de son maître et que Fleury subira des pertes considérables, occasionnées par des préparatifs inutiles ${ }^{35}$. Un tel sans-gêne fait murmurer. "Discours séditieux", grogne Vaudreuil; rien de plus insupportable que "l'esprit de mutinerie et d'indépendance" de ces perpétuels mécontents, les Canadiens ${ }^{36}$.

Avec les années, son caractère s'aigrit. Auprès de la Cour, il n'obtient plus toujours le même succès que naguère. Il sollicite, en 1717, le commandement des troupes en faveur de son fils aîné; il le redemande, en 1724, pour Cavagnial ${ }^{37}$. Il se heurte à deux refus. En 1718, il voudrait que Rigaud devînt lieutenant; Rigaud ne recevra sa promotion qu'en $1720^{38}$. Lui à qui le ministre pouvait autrefois reprocher "une espèce de mollesse" ${ }^{39}$, il se montre d'une sévérité excessive. Accusés d'avoir couru les bois, les fils du sieur de Coulonges comparaissent devant le Conseil Supérieur qui les renvoie, faute de preuves. "Cependant, raconte tranquillement Vaudreuil, je les ay toujours fait mettre en prison, j'espere que cette punition les rendra sage, et servira d'exemple aux autres"40. Il se brouille avec $\mathrm{Mgr}$ de Saint-Vallier et il n'a pas le beau rôle. Un jour, le marquis exige de la fabrique de Montréal qu'elle paie l'entretien du banc qu'il occupe à l'église; l'évêque s'y oppose et, en dépit "d'un grand: je le veux", Vaudreuil doit s'incliner ${ }^{41}$. A son tour, ce dernier reproche au prélat

35. Bégon à Maurepas, 10 juin 1725, AC, C 11A, 47: 142-154; copie d'une ordonnance de l'intendant Dupuy, 26 mars 1727, AC, C 11A, 49: 372-379; Beauharnais et Hocquart a Maurepas, 15 octobre 1730, AC, C 11A, 52: 54-56.

36. Vaudreuil à Maurepas, 18 mai 1725, AC, C 11A, 47: 81-82.

37. "Demandes d'emplois militaires au Canada," [ 1717], AC, C 11A, 37: 467468; Vaudreuil à Maurepas, 25 octobre 1724, AC, C 11A, 46: 83.

38. Vaudreuil au Conseil de Marine, 8 novembre 1718, AC, C 11A, 39: 137: le Conseil à Vaudreuil et à Bégon, 2 juin 1720, AC, B 42: 432v.

39. Pontchartrain à Vaudreuil, 9 juin 1706, AC, B 27: 223.

40. Vaudreuil a Maurepas, 25 octobre 1724, AC, C 11A, 46: 67.

41. RAPQ (1941-1942), 179. 
de marier sans permission des soldats et même des officiers; sur ce point, le Conseil de Marine soutient le gouverneur ${ }^{42}$. Mais $M$. de Québec riposte avec feu: "Le Conseil pourroit-il croire que devant en qualité de Pasteur remedier aux besoins spirituels de ses ouailles dont les soldats sont du nombre, quand on leur refusera impitoyablement pendant 8. ou 10. ans de se marier contre la volonté du Roy en leur laissant continuer leurs desordres et libertinages qui donnent a la Colonie une infinité d'enfans illegitimes,... que l'Eveque en fut quitte devant Dieu pour lui dire qu'il ne les a pas mariés parce que M. de Vaudreuil ne l'a pas voulu ?"43 En 1720, le gouverneur éclate: son propre neveu, Adhémar de Lantagnac, vient d'épouser "une fille sans bien et sans naissance $e^{44}$ dont il a veu la Mere servir chés son Pére qui tenoit cabaret", et l'évêque a autorisé cette mésalliance. Cette fois, la Cour blâme le prélat. Mais le mariage est fait et le jeune homme, exile ${ }^{45}$. Saint-Vallier attend son tour de marquer un point. L'occasion s'en présente bientôt: Vaudreuil, déclare le prélat, entre sans façon chez les religieuses, et jusque dans leur cloître, ce qui "enfle... le cœur et l'esprit de quelques-unes d'icelles" et attire au gouverneur une observation du Conseil de Marine ${ }^{46}$. Le marquis se fait encore dénoncer lorsqu'il lui prend fantaisie d'imposer aux Récollets de Québec un supérieur apparenté à sa femme, incident qui provoque d'âpres disputes chez les Pères: ce "scandale", lui écrit sèchement le ministre, ne serait pas arrivé "si vous aviés bien voulu ne pas vous mesler des choses qui ne vous regardent pas"47.

La marquise de Vaudreuil passe une première fois au Canada en $1721^{48}$, année où le gouverneur est promu grand-croix honoraire de

42. Vaudreuil au Conseil de Marine, 12 janvier 1719, AC, C 11A, 40: 94-95; délibération du Conseil de Marine sur une lettre de Vaudreuil du 20 octobre 1720, AC, C 11A, 43: 55-56.

43. Délibération du Conseil de Marine du 2 décembre 1721, ibid., 182.

44. Marie-Geneviève Martin de Lino, P.-G. Roy, La Famille Adhemar de Lantagnac (Lévis, 1908), 11.

45. Vaudreuil au Conseil de Marine, 20 octobre 1720, AC, C 11A, 43: 55; RAPQ (1941-1942), 200.

46. Saint-Vallier au Conseil de Marine, 1er octobre 1721, RAPQ (1941-1942), 209; le Conseil à Vaudreuil, 5 juin 1722, ibid., 214.

47. Maurepas à Vaưdreuil, 5 juin 1725, AC, B 48: 887 .

48. Le Conseil de Marine à Beauharnais, 27 avril 1721, AC, B 44: 153. Elle avait songé à y venir l'année précédente: $i d$. à $i d$., 17 avril 1720, ibid., $42: 168$. 
Saint-Louis ${ }^{49}$. Cette décoration le satisfait-elle? Sans doute, mais elle le laisse avec le désir du cordon bleu de l'ordre du Saint-Esprit ${ }^{50}$. Pendant son séjour, madame la "Gouvernante" parle beaucoup et inspire à Ramezay de graves appréhensions ${ }^{51}$. Elle ne manque d'ailleurs pas de vivacité: on se rappellera longtemps le déchaînement qu'elle a eu un jour contre l'ingénieur Chaussegros de Léry; l'algarade a fait sensation ${ }^{52}$. Madame de Vaudreuil revient dans la colonie en 1724. Partie de la Rochelle avec le successeur de Bégon, le pauvre M. Robert, qui meurt au début du voyage, elle arrive avec un chargement d'ardoise qu'elle a fait, naturellement, transporter aux frais de l'État pour sa maison de Montréal. Elle ne change guère. Tout en exprimant sa joie de retrouver le gouverneur "dans la plus parfaite santé du monde", elle déplore qu'il se soit fort endetté et fait sentir à Maurepas que sa famille a toujours besoin de protection $^{53}$. Comme d'habitude, le marquis descend à Montréal au printemps de 1725. Il y tombe malade au commencement de mai; au bout d'un mois, il se porte mieux, assure son médecin. Il a la force de rentrer à Québec, où il s'éteint le 10 octobre $^{54}$. Trois semaines plus tard, annonce-t-on, la marquise s'est embarquée à bord de la Reine des Anges ${ }^{55}$. Dès janvier 1726, la Cour lui accorde une pension de 3,000 livres sur les appointements du successeur de Vaudreuil, Beauharnais $^{56}$. Elle laisse au Canada deux filles, que Bégon amène en

49. A. Fauteux, Les Chevaliers de Saint-Louis en Canada, 93.

50. Maurepas à Mme de Vaudreuil, 5 juin 1725, AC, B 48: 874.

51. Ramezay à Maurepas, 16 octobre 1723, AC, C 11A, 45: 231-232; Ramezay à Vaudreuil, 16 octobre 1723, ibid., 235-237.

52. Maurrepas à Chazel, 29 mai 1725, AC, B 48: 838v-839.

53. Mme de Vaudreuil à Maurepas, 29 octobre 1724, AC, C 11A, 46: 106-115; Maurepas à Pajot, 21 avril 1724, AC, B 46: 434; Maurepas à Mme de Vaudreuil, 5 juin 1725, AC, B 48: 873 .

54. Bégon à Maurepas, 10 juin 1725, AC, C 11A, 47: 141; Longueuil et Bégon a Maurepes, 31 octobre 1725, ibid., 49; Les Ursulines de Québec, depuis leur établissement jusqu'd nos jours, 2: 140. L'acte de sépulture, du 13 octobre 1725, est reproduit dans Roy, 185. Le cœur du marquis fut envoyé en France et enterré dans le sépulcre des Vaudreuil: voir l'acte du 12 aout 1728 , reproduit $i b i d ., 48$, note 2.

55. Lơngueuil et Bégon à Maurepas, 31 octobre 1725, AC, C 11A, $47: 30$.

56. Maurepas à Mme de Vaudreuil, 11 janvier 1726, AC, B 49: 608; $i d$. à Beauharnais, 11 janvier 1726, ibid., 
1726 dans la métropole en allant prendre possession de son intendance du Havre ${ }^{57}$. En France, elle retrouve son fils aîné, LouisPhilippe, maintenant marié à une nièce d'Iberville; Philippe-Antoine, qui n'a pas reparu au Canada depuis qu'il a quitté "le parti de l'Église"; Jean, qui sert dans les Gardes Françaises, et JosephHyacinthe, qui s'en va tenter fortune aux Iles. La Nouvelle-France garde M. de Cavagnial, qui approche sérieusement de la trentaine, et Rigaud, soldat plein de talent, mais éclipsé par ses aînés. De Paris, où elle élit domicile, la grande dame ne se laisse pas perdre de vue à la Cour. Il lui faut surveiller ses intérêts. Sa maison de Montréal ne rapporte guère, elle offre de la vendre au roi. Qu'a-t-elle besoin de l'argent qu'elle en retirera? Elle fait vivre sa mère, ses filles, dont l'une est infirme, et une belle-sœur; elle soutient dans son emploi le fils qu'elle a aux Gardes Françaises, elle ne peut pas laisser sans secours ses autres enfants ${ }^{58}$. Elle entame au sujet de sa maison de patientes négociations avec la Cour: l'État refuse d'acquérir l'immeuble, mais il l'utilise et en verse un bon loyer ${ }^{59}$. La marquise ne pense pas seulement à elle-même. Il lui arrive de faire bénéficier ses lointains parents de sa recommandation ${ }^{60}$. Elle n'oublie pas les Canadiens: bienfaisante, elle recueille des fonds pour constituer une dot à la fille du lieutenant de roi des Trois-Rivières, désireuse d'entrer chez les Ursulines ${ }^{61}$. Elle entretient ainsi la popularité du nom de Vaudreuil.

Cette popularité durera. Au fond, n'est-elle pas méritée ? Sans toujours donner le bon exemple à son fils, qui apprend auprès de lui l'art de gouverner, le marquis est parvenu à faire traverser au Canada

57. Maurepas à Bégon, 14 mai 1726, AC, B 49: 673-673v; id. à id., 3 décembre 1726, ibid., 282.

58. Mme de Vaudreuil à Maurepas, 1727, AC, C 11A, 49: 403-404.

59. Maurepas à Beauharnais et à Dupuy, 29 avril 1727, AC, B 50: 510-510v; Maurepas à Dupuy, 29 avril 1727, ibid., 510v-511; id à $i d$., 24 mai 1728, AC, B 52: 533v; copie du bail du 20 aout 1728, AC, C 11A, 73: 19-21; Daigremont à Maurepas, 15 octobre 1728, AC, C 11A, 50: 143; Maurepas à Hocquart, 2 mai 1729, AC, B 53: 533v-534; Hocquart à Maurepas, 25 octobre 1729, AC, C 11A, 51: 253; Maurepas à Longueuil, 28 mars 1730, AC, B 54: 417v.

60. Mme de Vaưdreuil a Maurepas, 1727, AC, C 11A, 49: 405-406. Voir lettre du chanoine Hazeur, 1731, BRH, 16 (1910): 194.

61. Maưrepas à Beauharnais, 24 avril 1731, AC, B 55: 498. 
les années sombres de la guerre et les années instables de l'aprèsguerre. Au lendemain de la paix d'Utrecht, il a mis le pays en bonne voie de redressement. Dès 1716 , s'ébauche un vaste programme de relèvement économique. Les réalisations ne tardent pas. Des débouchés s'offrent tout de suite aux Antilles et un marché se présente bientôt à Louisbourg pour les céréales canadiennes; ainsi stimulée, l'agriculture produit et les prix sont bons. La colonie trouve aussi à vendre du bois: ce n'est pas par hasard que, de 1719 à 1721, le nombre des scieries passe de dix-neuf à trente. Bien que l'effondrement de la Compagnie de la Colonie ait failli écraser les commerçants de fourrures, ceux-ci trouvent dans une contrebande massive le moyen de se récupérer d'une partie de leurs pertes ${ }^{62}$. Si les données démographiques indiquent quelque chose, elles laissent deviner un courant de prospérité: pendant le premier quart du XVIIIe siècle, la population double, et ce gain s'effectue presque en entier après $1713^{63}$. Douze ans de paix et de bien-être grandissant, que veut-on de plus pour expliquer la renommée de Vaudreuil, la légende de Vaudreuil? S'il leur en fallait davantage, les Canadiens n'auraient qu'à se rappeler qu'en 1714 le débonnaire marquis a détourné la Cour du projet de les charger d'impôts comme des Français ${ }^{64}$. Le gouverneur sait se faire aimer du grand nombre. Bien que sujet à des sautes d'humeur et à quelque rancune ${ }^{65}$, il possède un caractère d'une grande aménité. Toute sa correspondance est celle d'un homme attentif à se dominer: sauf peut-être dans les dernières années, sa modération, même lorsqu'il écrit pour se défendre, contraste avec la violence de ses rares adversaires. Sans se révéler sensible, - la mode n'en viendra que plus tard, - il s'apitoie sur le sort des pauvres $^{66}$. Les traitants se plaignent de lui. Ils se défendent, ils ont raison. Mais que font au juste ses agents? Cherchent-ils à mettre sur pied un monopole des fourrures semblable à celui des denrées ali-

62. La Civilisation de la Nouvelle-France, 95, 97, 103, 105, 109.

63. Ibid., 26; 39.

64. Ibid., 77-79.

65. Pontchartrain à Vaudreuil, 10 mai 1710, RAPQ (1946-1947), 373; Vaudreuil à Pontchartrain, 25 octobre 1710, ibid., 385; Pontchartrain à Vaudreuil, 7 juillet 1711, ibid., 415, 417.

66. La Civilisation de la Nouvelle-France, 70. 
mentaires que Bégon tente de constituer? Non; ils exploitent deux postes. Vaudreuil n'accapare pas la traite, il s'efforce d'en partager les profits. Trône-t-il, comme plus tard Bigot, au sommet d'une insolente fortune et s'enrichit-il de la misère du pays? Au contraire, il prend sa part d'une prospérité dont il est, dans une bonne mesure, l'artisan et il n'accumule point: il vit selon sa condition et installe ses fils dans les emplois de l'État. A la fin, c'est un vieux gentilhomme qui se survit, entouré de ses vieilleries. Dans la cuisine du château Saint-Louis, traîne un four de campagne de cuivre rouge, coiffé d'un couvercle de tôle, souvenir du temps où le cadet de Gascogne allait en expédition; aux murs de la grande chambre, pendent un miroir à cadre doré, une tapisserie de Flandre et un portrait de Louis XIV; dans le garde-meuble, parmi les serviettes, les nappes, les cravates de mousseline, voilà trois anciens bonnets de coton tricoté, estimés trente-six sous; ailleurs, dort un tableau où se figent des seigneurs de la cour et de la lignée... ${ }^{67} \cdot \mathrm{Au}$ milieu de ces objets, le gouverneur général a vécu entouré d'honneurs, affamé de distinctions, assiégé par les soucis d'une famille dispersée, compagnon de la pensée de l'épouse lointaine et pratique qui prépare la carrière des fils, incrustés déjà dans le service du Roy.

Guy Fregault, professeur à la Faculté des Lettres, Université de Montreal

67. "Inventaire et description" des biens de Vaudreuil, 19-25 juin 1726, RAPQ (1921-1922), 238-257. 